\title{
Online Healthcare Service Delivery in Nepal during COVID Era: A challenge
}

\section{Sharma P}

WHO declared COVID-19 as pandemic on 11th March 2020. Preventive strategy like social distancing was enforced by governments across the globe. To address this crisis, Nepal government implemented nationwide lockdown from 24th March 2020 following the announcement of the second positive case of COVID-19. In the beginning, as there was shut down of the outpatient department, many problems followed this crisis, especially in health care service delivery. Access to health care center was already a problem in normal times because of the topography and bad roadway conditions of our country. This was further added up by this adverse situation. Had our online health care service been stronger, this situation could have been easily tackled. This was the right time to practice Telemedicine including teleradiology and teledermatology for optimum benefit.

Telemedicine can be used for awareness, diagnosing, treating, and controlling diseases during COVID-19 outbreak. To some extent, Teledermatology, either real time or store and forward type, is already in practice in Nepal to help patients in the rural areas during normal times. But this is the time to highlight its benefit in healthcare delivery while still practicing social distancing. Teleconsultations were practiced during this pandemic. social media like viber, whatsapp, messenger was used for free consultation. Clinicians also provided their private numbers for teleconsultation for the needy people.

Telemedicine is the use of electronic information and telecommunication technology to get the health care one requires. This would demand high speed internet access even in the rural areas. Telecommunication can be performed by a mobile device or a computer with internet connections. This has become easy to people in the hilly and mountainous regions due to developed mobile services by using data packs in the country even when there are no Wi-Fi systems.

COVID 19 is a public health emergency with international concern. However, other ailments with equal medical concern still persists. This is the time to promote telemedicine which is the only tool at the present time to deliver health care practice maintaining the social distancing. But it remains a challenge. The government should also invest and make policies regarding the practice of telemedicine. 